\title{
Eldecalcitol is more effective for the prevention of osteoporotic fractures than alfacalcidol
}

\author{
Toshitaka Nakamura $\cdot$ Toshiyuki Takano • \\ Masao Fukunaga $\cdot$ Masataka Shiraki • \\ Toshio Matsumoto
}

Received: 29 August 2012 / Accepted: 16 December 2012/Published online: 11 April 2013

(C) The Japanese Society for Bone and Mineral Research and Springer Japan 2013

\begin{abstract}
Eldecalcitol, a vitamin $\mathrm{D}_{3}$ analogue, significantly reduces the risk of new vertebral fractures and increases bone mineral density (BMD) more than does alfacalcidol. To determine the effect of eldecalcitol on the incidence of all fragility fractures caused by osteoporosis, we conducted post hoc analyses of the phase III clinical trial to evaluate the incidence of the osteoporotic fractures defined in the World Health Organization (WHO) Technical Report, and, also, the incidence of the major osteoporotic fractures utilized in the WHO Fracture Risk Assessment Tool (FRAX), and compared those in the eldecalcitol group with those in the alfacalcidol group. We also analyzed the incidence of osteoporotic fractures stratified by prespecified risk factors for fractures. Eldecalcitol treatment reduced the incidence of osteoporotic fractures defined by the WHO more than alfacalcidol treatment (18.6\% vs. $25.2 \%$; hazard ratio, 0.70; $95 \% \mathrm{CI}$, 0.54-0.93). Prevalent vertebral fractures, two or more
\end{abstract}

T. Nakamura $(\square)$

National Center for Global Health and Medicine, 1-21-1

Toyama, Shinjuku-ku, Tokyo 162-8655, Japan

e-mail: tnakamura@hosp.ncgm.go.jp

T. Takano

Chugai Pharmaceutical Co. Ltd., Tokyo 103-8324, Japan

M. Fukunaga

Kawasaki Medical School, Okayama 701-0192, Japan

M. Shiraki

Research Institute and Practice for Involutional Diseases,

Nagano 399-8101, Japan

T. Matsumoto

University of Tokushima Graduate School of Medical Sciences,

Tokushima 770-8503, Japan prevalent vertebral fractures, and total hip BMD $T$ score less than -2.5 were the risk factors for new osteoporotic fractures with significant differences between the two treatments. Eldecalcitol also decreased the incidence of major osteoporotic fractures in the FRAX more than alfacalcidol (11.1\% vs. $16.3 \%$; hazard ratio, 0.66; $95 \% \mathrm{CI}$, 0.46-0.94). In conclusion, treatment with eldecalcitol reduced the risk of fragility fractures caused by osteoporosis compared with alfacalcidol administration, which may result from a potent effect of eldecalcitol on BMD, bone structure, and bone turnover.

Keywords Eldecalcitol · Fracture - FRAX . Osteoporosis · Vitamin D

\section{Introduction}

Eldecalcitol is a new active vitamin $\mathrm{D}_{3}$ analogue with a hydroxypropyloxy group introduced at the $2 \beta$ position of $1 \alpha, 25$-dihydroxyvitamin $\mathrm{D}_{3}$. Alfacalcidol, $1 \alpha$-hydroxyvitamin $D_{3}$, is a prodrug of an active form of vitamin $\mathrm{D}_{3}$. A randomized, double-blind, placebo-controlled, clinical study demonstrated that, compared to a placebo, 1-year treatment with eldecalcitol significantly increased lumbar spine and total hip bone mineral density (BMD) in a dose-dependent manner [1]. Further, in comparison to alfacalcidol treatment, eldecalcitol treatment significantly decreased the incidences of vertebral fractures (morphometric and clinical) and wrist fractures, with marked increases in lumbar spine BMD and total hip $\mathrm{BMD}$, as assessed by a 3-year, randomized, doubleblind, clinical trial [2]. On the basis of these results, eldecalcitol has been approved for the treatment of osteoporosis in Japan. 
A characteristic type of fracture frequently found in osteoporosis is the so-called fragility fracture. However, diagnosis of fragility fractures in patients with osteoporosis is often difficult in daily practice because of uncertainty in evaluating the degree of causative energy loaded onto the fracture site. Recently, the World Health Organization (WHO) Scientific Group [3, 4] introduced a new approach to characterizing fractures relevant to osteoporosis. These "osteoporotic fractures" are fractures whose risk of incidence is associated with low bone mass and whose incidences rise with age after the age of 50 years. Fractures pertinent to these criteria are those at the spine, distal forearm (wrist), humerus, ribs, clavicle/scapula/sternum, pelvis, tibia/fibula, hip, and other femoral fractures. Prevention of these osteoporotic fractures is of prime importance in patients with osteoporosis. We have already reported the reduction in the incidence of three major predefined nonvertebral fractures of the humerus, wrist, and hip [2]. However, we did not analyze the incidences of osteoporotic fractures including major fractures used in the WHO Fracture Risk Assessment Tool (FRAX).

In the present study, we analyzed the data derived from the phase III clinical trial of the efficacy of eldecalcitol versus alfacalcidol on fracture prevention in osteoporotic patients (Clinical Trials Gov Number NCT00144456) to determine the effect of eldecalcitol on the incidence of osteoporotic fractures in comparison to that of alfacalcidol. We also analyzed the effect of each agent on the risk of major osteoporotic fractures included in FRAX-i.e., clinical vertebral fracture and fractures of the hip, distal forearm, and humerus-without discriminating between traumatic and nontraumatic fractures. Then, we analyzed the incidences of osteoporotic fractures stratified by prespecified risk factors for fractures at baseline.

\section{Materials and methods}

Details of the double-blind fracture prevention clinical study of eldecalcitol have been published previously [2]. Briefly, 1,054 patients with primary osteoporosis [5, 6] were divided into two groups: an eldecalcitol group $(n=528)$ and an alfacalcidol group $(n=526)$. They were given either oral eldecalcitol $(0.75 \mu \mathrm{g})$ or oral alfacalcidol $(1.0 \mu \mathrm{g})$ once a day for 3 years (36 months). Patients with serum 25-hydroxyvitamin $\mathrm{D}$ values lower than $20 \mathrm{ng} / \mathrm{ml}$ at the time of enrollment were given an oral vitamin $D_{3}$ supplement (400 IU) once a day without calcium supplementation. Patients with lumbar spine or total hip BMD $T$ score below -1.7 were enrolled if they had one to five vertebral fractures. Patients without vertebral fractures were also enrolled if their lumbar spine or total hip BMD $T$ score was below -2.6 and they were aged 70 years or older, or if their $T$ score was below -3.4 and they were aged less than 70 years. Patients with metabolic bone disease such as primary hyperparathyroidism, Cushing's syndrome, premature menopause, poorly controlled diabetes mellitus (HbA1c $>9 \%$ ), or other causes of secondary osteoporosis or patients who had a history of urolithiasis were excluded from the study. Patients were also excluded if they had taken any oral bisphosphonates within 6 months before entry or for more than 2 weeks during the period 6-12 months before entry, or intravenous bisphosphonates at any time; had taken glucocorticoids, calcitonin, vitamin $\mathrm{K}_{2}$, active vitamin $\mathrm{D}$ compounds, raloxifene, or hormone replacement therapy within 2 months; had serum calcium levels above $10.4 \mathrm{mg} / \mathrm{dl}$ or urinary calcium excretion greater than $0.4 \mathrm{mg} / \mathrm{dl} \mathrm{GF}$; or had serum creatinine above $1.3 \mathrm{mg} / \mathrm{dl}$.

The primary endpoint of the study was the incidence of nontraumatic new vertebral fractures, and the secondary endpoints were the percent change in lumbar spine BMD and total hip BMD, percent change of bone turnover markers, and incidence of nonvertebral fractures. The incidence of nontraumatic new vertebral fractures was evaluated by using lateral radiographs of the thoracic and lumbar spine obtained at baseline and at 6, 12, 24, and 36 months after initiation of drug administration. Incidences of nonvertebral fractures were assessed using radiographic examinations at the clinical site by investigators, and the data were reported at the time of incidence to the central facility and analyzed. In this study, we compared the two treatment groups with respect to the incidence of osteoporotic fractures as defined by the WHO Scientific Group [3, 4], their site-specific incidences, and the incidence of major osteoporotic fractures included in FRAX. We also analyzed the incidence of osteoporotic fractures based on prespecified risk factors for fractures at baseline: age, serum 25-hydroxyvitamin D value, number and degree of severity of prevalent vertebral fractures, lumbar spine BMD, and total hip BMD.

\section{Statistical analysis}

We performed the analysis for all subjects in the Full Analysis Set (eldecalcitol group, $n=526$; alfacalcidol group, $n=523$ ). First, the incidences of fractures at any of the nine osteoporotic fracture sites and their subgroup analyses were evaluated by Kaplan-Meier estimation. Then, the incidences of fractures at each respective site were assessed. The incidences of major osteoporotic fractures were also estimated by the Kaplan-Meier method. To compare the effects of eldecalcitol with those of alfacalcidol, we performed stratified log-rank tests (two-sided, $5 \%$ significance level) and determined the hazard ratios and the $95 \%$ confidence intervals of those incidences by 
stratified Cox regression models, as described in the subgroup analyses of the previous report [2], with adjustment for the number of prevalent vertebral fractures at baseline (categories were no fracture, one fracture, or more than one fracture).

\section{Results}

There were no significant differences in the patients' background data with regard to age, percentage of male patients, body mass index, the prevalence of vertebral fractures, nonvertebral fractures, BMD, or metabolic bone markers (Table 1).

The incidence of all osteoporotic fractures for 3 years was $18.6 \%$ in the eldecalcitol group and $25.2 \%$ in the alfacalcidol group, according to the Kaplan-Meier estimate (Fig. 1a), and the risk of osteoporotic fracture in the eldecalcitol group was significantly lower than in the alfacalcidol group (hazard ratio, 0.70; $95 \% \mathrm{CI}, 0.54-0.93$; $p=0.013$ ). The incidence of nonvertebral osteoporotic fractures was $5.8 \%$ in the eldecalcitol group and $9.7 \%$ in the alfacalcidol group (Fig. 1b), indicating a significant

Table 1 Baseline characteristics of enrolled patients

\begin{tabular}{lll}
\hline & $\begin{array}{l}\text { Eldecalcitol } \\
(n=526)\end{array}$ & $\begin{array}{l}\text { Alfacalcidol } \\
(n=523)\end{array}$ \\
\hline Age (years) & $72.2 \pm 6.59$ & $72.1 \pm 6.62$ \\
Male patients $[n(\%)]$ & $9(1.71)$ & $15(2.87)$ \\
Body mass index $\left(\mathrm{kg} / \mathrm{m}^{2}\right)$ & $22.3 \pm 3.19$ & $22.3 \pm 3.20$ \\
Number of prevalent vertebral & $1.19 \pm 1.28$ & $1.25 \pm 1.36$ \\
fractures & $198(37.6)$ & $193(36.9)$ \\
0 [n(\%)] & $155(29.5)$ & $159(30.4)$ \\
1 [ $(\%)]$ & $173(32.9)$ & $171(32.7)$ \\
$\geq 2[n(\%)]$ & & \\
Prevalent vertebral fracture & $198(37.6)$ & $193(36.9)$ \\
Grade 0 $[n(\%)]$ & $110(20.9)$ & $118(22.6)$ \\
Grade 1 $[n(\%)]$ & $132(25.1)$ & $102(19.5)$ \\
Grade 2 $[n(\%)]$ & $86(16.3)$ & $110(21.0)$ \\
Grade 3 $[n(\%)]$ & & \\
History of nonvertebral fracture & $367(69.8)$ & $379(72.5)$ \\
Absent $[n(\%)]$ & $159(30.2)$ & $144(27.5)$ \\
Present $[n(\%)]$ & $-2.70 \pm 0.94$ & $-2.72 \pm 0.90$ \\
Lumbar spine bone mineral & & \\
density (BMD) $T$ score & $-2.26 \pm 0.82$ & $-2.27 \pm 0.79$ \\
Total hip BMD $T$ score & $33.4 \pm 14.4$ & $33.8 \pm 12.5$ \\
Bone alkaline phosphatase & & \\
(BAP) $(\mathrm{U} / \mathrm{l})$ & $58.0 \pm 58.7$ & $57.0 \pm 32.7$ \\
Urinary NTx (nmol BCE/mmol Cr) & & \\
\hline
\end{tabular}

Data are mean \pm SD. There were no significant differences in the patients' background data decrease in the risk in the eldecalcitol group relative to that in the alfacalcidol group (hazard ratio, 0.59; $95 \% \mathrm{CI}$, 0.37-0.94, $p=0.026$ ).

The incidence of the four major osteoporotic clinical fractures used in FRAX (clinical vertebral fractures, and fractures of the humerus, distal forearm, and hip) for 3 years was $11.1 \%$ in the eldecalcitol group and $16.3 \%$ in the alfacalcidol group. The risk of these fractures was significantly lower in the eldecalcitol group than in the alfacalcidol group (hazard ratio, 0.66; $95 \% \mathrm{CI}, 0.46-0.94$; $p=0.020$ ) (Fig. 2).

In the subgroup analyses of osteoporotic fractures, all the values of the point estimates for hazard ratio in the eldecalcitol group were less than 1.0 compared to the alfacalcidol group. The categories of subgroups that showed marked significant differences $(p<0.01)$ were presence of prevalent vertebral fractures (hazard ratio, 0.66 ; $95 \%$ CI, $0.49-0.89$; $p=0.007$ ), two or more prevalent vertebral fractures (hazard ratio, 0.59; $95 \% \mathrm{CI}$, $0.41-0.86 ; p=0.006$ ), and total hip BMD T-score of less than -2.5 (hazard ratio, $0.55 ; 95 \% \mathrm{CI}, 0.36-0.84$; $p=0.006$ ) (Fig. 3).

Site-specific numbers of osteoporotic fractures of the vertebrae, ribs, clavicle/scapula/sternum, tibia/fibula, and distal forearm that occurred during the 3-year trial were smaller in the eldecalcitol group than in the alfacalcidol group, but a statistically significant difference was observed only for vertebra and distal forearm (vertebra: hazard ratio, 0.72; $95 \% \mathrm{CI}, 0.52-0.99$; distal forearm: hazard ratio, $0.28 ; 95 \%$ CI, 0.11-0.69) (Table 2).

\section{Discussion}

This study clearly demonstrated that, compared with $1.0 \mu \mathrm{g}$ daily alfacalcidol administration, daily administration of $0.75 \mu \mathrm{g}$ eldecalcitol reduced the risk of osteoporotic fractures as defined by WHO and decreased the risk of major osteoporotic fractures included in FRAX. A significant decrease in the incidence of nonvertebral osteoporotic fractures was also observed in the eldecalcitol group.

In the subgroup analysis, eldecalcitol was found to be more effective at reducing the risk of osteoporotic fractures than alfacalcidol in patients with total hip BMD $T$ score less than -2.5 . The hazard ratio for patients with two or more prevalent vertebral fractures was markedly smaller than the hazard ratios for patients with one or no prevalent vertebral fracture. Thus, eldecalcitol seems to be more effective than alfacalcidol in preventing osteoporotic fractures in patients with multiple prevalent vertebral fractures. 
Fig. 1 Kaplan-Meier estimates osteoporotic fractures defined by the WHO Scientific Group (a), and the incidence of nonvertebral osteoporotic fractures in the eldecalcitol and alfacalcidol groups (b)

Fig. 2 Kaplan-Meier estimates of the incidence of major osteoporotic fractures included Tool (FRAX) in the eldecalcitol and alfacalcidol groups of the incidence of all in the Fracture Risk Assessment

a

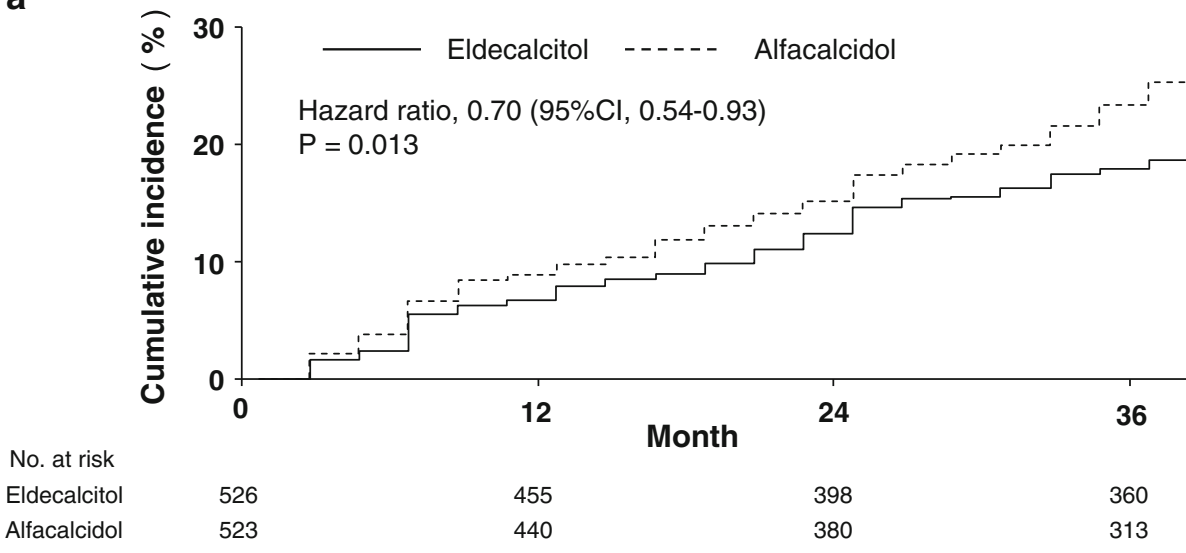

b
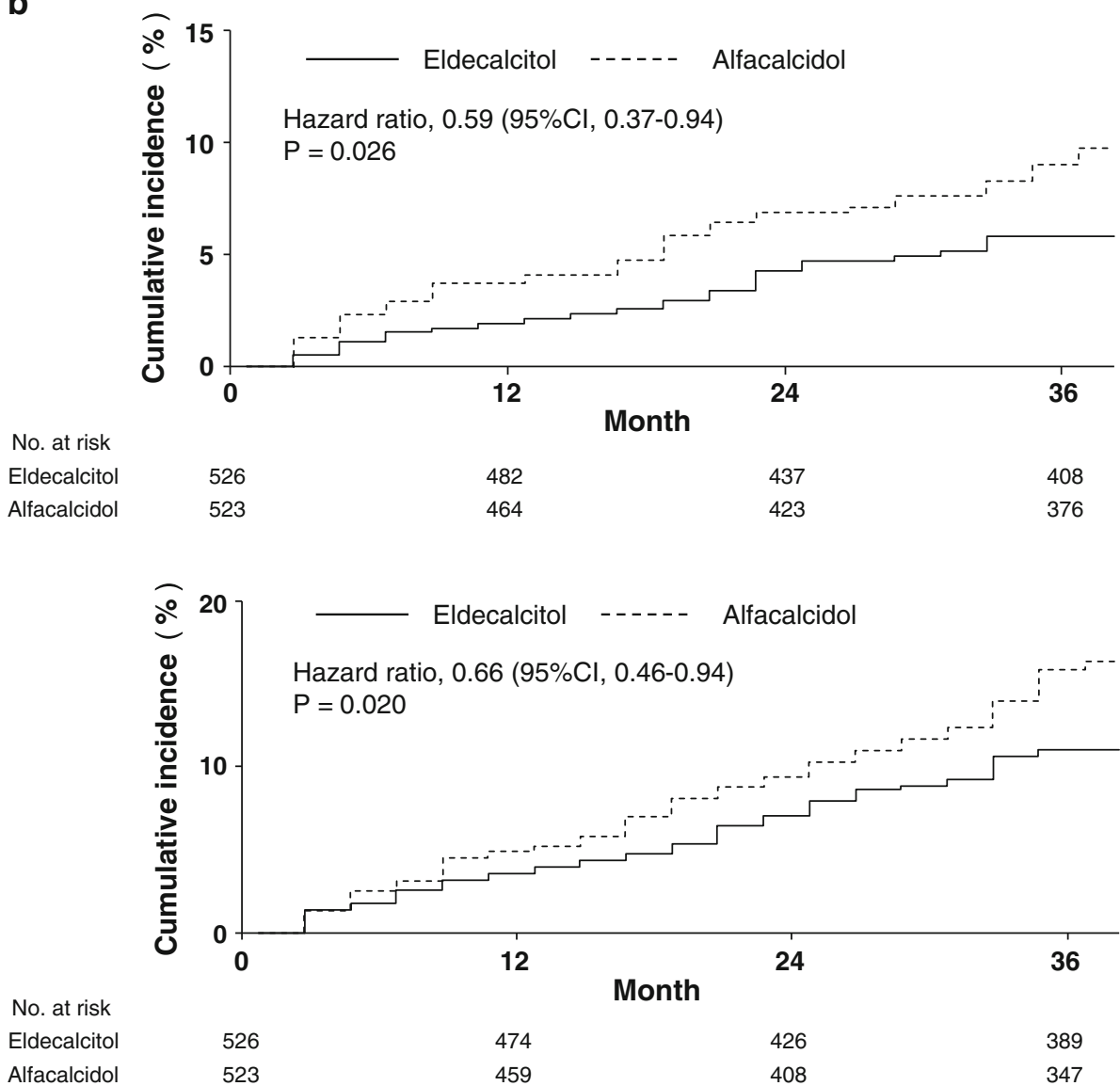

In the previous study, we demonstrated the efficacy of eldecalcitol in preventing morphometric vertebral fractures and nonvertebral fragility fractures at three major sites (humerus, wrist, and hip) [2]. In this study, we analyzed the incidences of all radiographically confirmed osteoporotic fractures of the vertebrae, ribs, pelvis, humerus, clavicle/ scapula/sternum, hip, other femoral sites, tibia/fibula, and distal forearm. Then, we compared the incidences of the nonvertebral osteoporotic fractures. We also calculated the incidences of the four major osteoporotic fractures listed in

the FRAX [vertebrae (clinical fracture), hip, distal forearm, and humerus]. These data compatibly indicated the superior efficacy of eldecalcitol compared to that of alfacalcidol on the prevention of fractures in patients with osteoporosis. The superiority of eldecalcitol in preventing nonvertebral osteoporotic fractures appeared to be largely the result of reductions in the incidence of fractures of the ribs, clavicle/ scapula/sternum, tibia/fibula, and distal forearm.

The effect of eldecalcitol on reducing the risk of osteoporotic fractures and nonvertebral osteoporotic fractures 


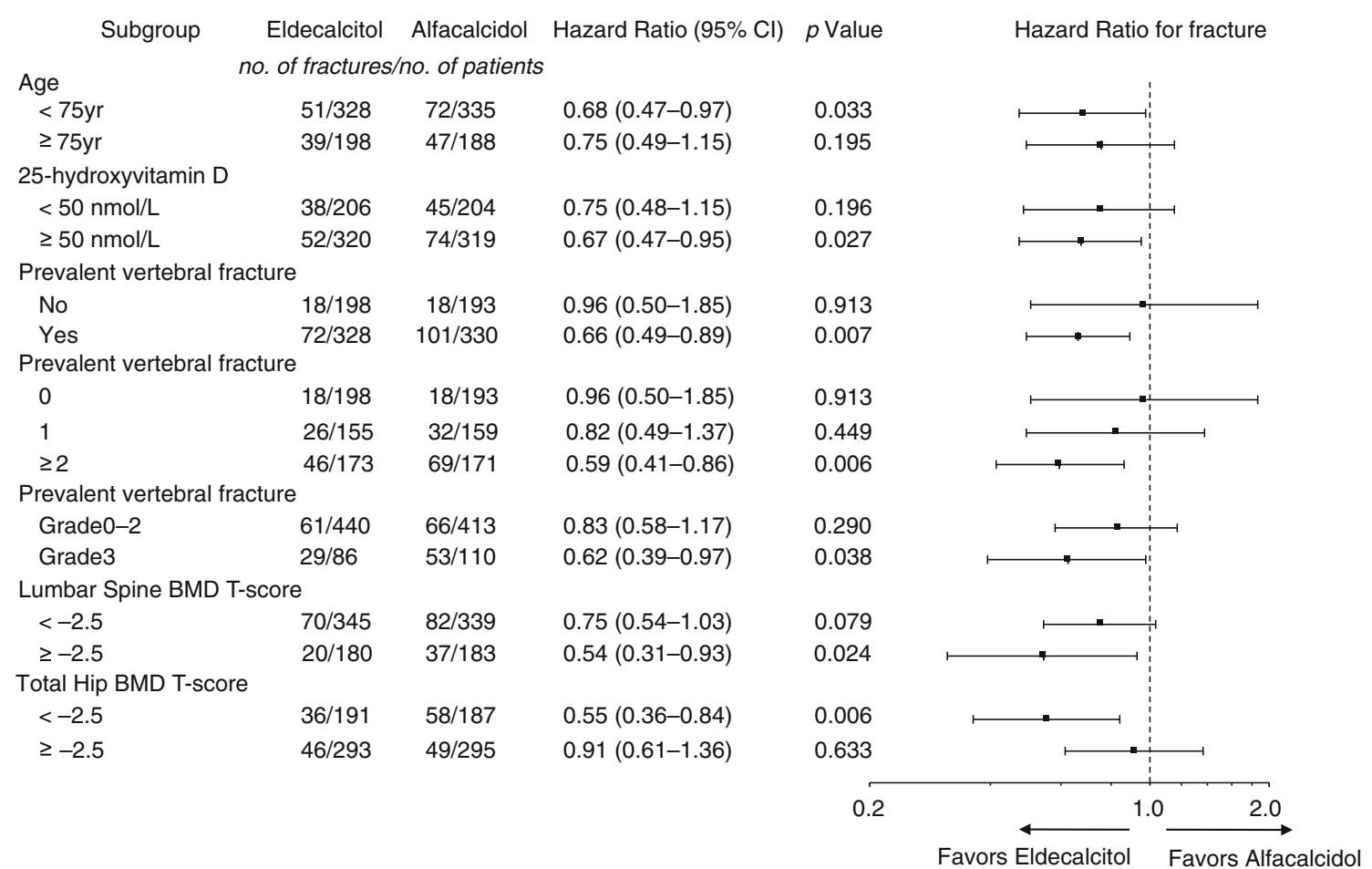

Fig. 3 Subgroup analyses of the effect of eldecalcitol in comparison with alfacalcidol on osteoporotic fractures

Table 2 Osteoporotic fractures defined by the WHO Scientific Group and their site-specific incidences a Tibial and fibular fractures listed are those in women

\begin{tabular}{llll}
\hline & $\begin{array}{l}\text { Eldecalcitol } \\
(n=526)\end{array}$ & $\begin{array}{l}\text { Alfacalcidol } \\
(n=523)\end{array}$ & $\begin{array}{l}\text { Hazard ratio } \\
(95 \% \text { CI })\end{array}$ \\
\hline $\begin{array}{l}\text { All osteoporotic fractures } \\
\text { Nonvertebral osteoporotic fractures }\end{array}$ & 28 & 119 & $0.70(0.54-0.93)$ \\
Osteoporotic fractures at specific sites & & 46 & $0.59(0.37-0.94)$ \\
$\quad$ Vertebra & 64 & & \\
Ribs & 8 & 83 & $0.72(0.52-0.99)$ \\
Pelvis & 3 & 14 & $0.56(0.23-1.33)$ \\
Humerus & 2 & 1 & $2.95(0.31-28.34)$ \\
Clavicle, scapula, sternum & 1 & 3 & $1.92(0.17-21.22)$ \\
Hip & 7 & 5 & $0.33(0.03-3.20)$ \\
Other femoral fracture & 0 & 1 & $1.41(0.45-4.44)$ \\
Tibia, fibula & 1 & 5 & - \\
Distal forearm & 6 & 21 & $0.20(0.02-1.69)$ \\
\hline
\end{tabular}

seems to depend on the potent effect of eldecalcitol in increasing BMD and strengthening bone structure. Eldecalcitol was shown to increase lumbar BMD by $3.3 \%$ and total hip BMD by $1.5 \%$ in 1 year in comparison to placebo [1] and to increase lumbar BMD by $3.3 \%$ and total hip BMD by $2.7 \%$ in 3 years compared to alfacalcidol [2]. Subgroup analysis in this study confirmed the potent efficacy of eldecalcitol compared to alfacalcidol. With eldecalcitol treatment, urinary NTx, a bone resorption marker, was observed to decrease by $20 \%$ in 3 months in comparison to baseline levels [1] and by $23 \%$ in 3 years compared to that when treated with alfacalcidol [2]. Also, femoral bone geometry assessment using clinical computed tomography (multidetector-row CT) scanning has shown that, compared with alfacalcidol, eldecalcitol more markedly increases the cross-sectional cortical bone area in patients with osteoporosis, maintains the thickness of cortical bone, and improves the bone biomechanical parameters of the femoral neck [7]. The volumetric BMD value at the femoral neck also significantly increased with eldecalcitol treatment compared to with alfacalcidol treatment. These data strongly suggest that eldecalcitol improves the 
material strength of bone and also the cortical bone structure.

Preventing a chain reaction of fragility fractures in patients with osteoporosis is critically important in daily clinical practice. Meta-analysis studies suggest that a previous fracture history is associated with a significant (approximately doubled) increase in risk of any fracture compared with individuals without a prior fracture [8, 9]. Robinson et al. [10] reported that fractures of the hip, wrist, and proximal part of humerus were associated with a high risk of later re-fracture at any of the four sites they examined (hip, wrist, proximal part of the humerus, and ankle), and the relative risks were 5.76, 3.98, and 4.87, respectively. Center et al. [11] reported that absolute risk of subsequent fracture was increased in women across all age groups for most fracture types with clinical symptoms, including hip, vertebra, upper limb, ribs, and lower limb, and their relative risks were 2.79, 2.52, 1.69, 1.84, and 1.39, respectively. These data indicate that a prevalent fracture is a strong indicator of secondary fractures. This study confirmed the potent efficacy of eldecalcitol in preventing subsequent osteoporotic fractures in patients with prevalent, especially multiply prevalent, vertebral fractures.

This study has several limitations. First, this was a post hoc analysis. Second, this study had no placebo group. Third, the study population was not large enough to reliably evaluate the subgroup analyses and analyses of sitespecific osteoporotic fractures. Therefore, these analyses should be considered exploratory.

In conclusion, daily administration of $0.75 \mu \mathrm{g}$ eldecalcitol reduces the risk of osteoporotic fractures compared with $1.0 \mu \mathrm{g}$ alfacalcidol administration in 3 years. Eldecalcitol was also superior to alfacalcidol in reducing nonvertebral osteoporotic fractures, which could be the result of the potent effect that eldecalcitol has on BMD, bone structure, and bone turnover.

Acknowledgments The present study was sponsored by Chugai Pharmaceutical Co., Ltd. The sponsor of the study participated in the design of the study, data collection, data analyses, data interpretation, and writing of the report. The sponsor supplied the study medication and had responsibility for data collection and quality control. The corresponding author had full access to all the data in the study and had responsibility for the decision to submit for publication. The authors thank all the investigators who participated in the eldecalcitol phase III clinical trial.

Conflict of interest T.N. has received consulting fees (Asahi Kasei Pharma, Astellas, Banyu, Chugai, Daiichi Sankyo, Eisai, Eli Lilly Japan, Ono, Takeda, Teijin Pharma) and belongs to the Japan
Ministry of Health, Welfare and Labour as a councilor for hospital administration and social medical insurance. T.T. is a full-time employee of Chugai Pharmaceutical Co., Ltd. M.F. is a member of an advisory council (Asahi Kasei Pharma, Astellas). M.S. has received consulting fees (Asahi Kasei Pharma, Astellas, Chugai, Daiichi Sankyo, MSD, Teijin Pharma) and lecture fees (Eisai, Ono). T.M. is a member of an advisory board (Lilly) and has received consulting fees (Asahi Kasei Pharma, Astellas, Chugai, Daiichi Sankyo, Ono, Teijin Pharma).

\section{References}

1. Matsumoto T, Miki T, Hagino H, Sugimoto T, Okamoto S, Hirota T, Tanigawara Y, Hayashi Y, Fukunaga M, Shiraki M, Nakamura $\mathrm{T}$ (2005) A new active vitamin D, ED-71, increases bone mass in osteoporotic patients under vitamin D supplementation: a randomized, double-blind, placebo-controlled clinical trial. J Clin Endocrinol Metab 90:5031-5036

2. Matsumoto T, Ito M, Hayashi Y, Hirota T, Tanigawara Y, Sone T, Fukunaga M, Shiraki M, Nakamura T (2011) A new active vitamin $\mathrm{D}_{3}$ analog, eldecalcitol, prevents the risk of osteoporotic fractures: a randomized, active comparator, double-blind study. Bone (NY) 49:605-612

3. Kanis JA, on behalf of the World Health Organisation Scientific Group (2007) Assessment of osteoporosis at the primary health care level. WHO Collaborating Centre for Metabolic Bone Diseases, University of Sheffield

4. Kanis JA, Oden A, Johnell O, Jonsson B, De Laet C, Dawson A (2001) The burden of osteoporotic fractures: a method for setting intervention thresholds. Osteoporos Int 12:417-427

5. Raisz LG (2008) Overview of pathogenesis. In: Rosen CJ (ed) Primer on the metabolic bone diseases and disorders of mineral metabolism, 7th edn. American Society for Bone and Mineral Research, Washington, DC, pp 203-206

6. Raisz LG (2005) Pathogenesis of osteoporosis: concepts, conflicts, and prospects. J Clin Invest 115:3318-3325

7. Ito $M$, Nakamura $T$, Fukunaga $M$, Shiraki $M$, Matsumoto $T$ (2011) Effect of eldecalcitol, an active vitamin D analog, on hip structure and biomechanical properties: 3D assessment by clinical CT. Bone (NY) 49:328-334

8. Klotzbuecher CM, Ross PD, Landsman PB, Abbott TA III, Berger M (2000) Patients with prior fractures have an increased risk of future fractures: a summary of the literature and statistical synthesis. J Bone Miner Res 4:721-739

9. Kanis JA, Johnell O, De Laet C, Johansson H, Oden A, Delmas P, Eisman J, Fujiwara S, Garnero P, Kroger H, McCloskey EV, Mellstrom D, Melton LJ, Pols H, Reeve J, Silman A, Tenenhouse A (2004) A meta-analysis of previous fracture and subsequent fracture risk. Bone (NY) 35:375-382

10. Robinson CM, Royds M, Abraham A, McQueen MM, CourtBrown CM, Christie J (2002) Refractures in patients at least forty-five years old: a prospective analysis of twenty-two thousand and sixty patients. J Bone Joint Surg Am 84A:1528-1533

11. Center JR, Bliuc D, Nguyen TV, Eisman JA (2007) Risk of subsequent fracture after low-trauma fracture in men and women. JAMA 297:387-394 